\title{
Kinetics of initiation of the third generation Grubbs metathesis catalyst: convergent associative and dissociative pathways
}

\author{
Veronica Forcina, Andrés García-Domínguez and Guy C. Lloyd-Jones (D) *
}

Received 23rd April 2019, Accepted 21st May 2019

DOI: $10.1039 / c 9 f d 00043 g$

The kinetics of the nominally irreversible reaction of the third generation Grubbs catalyst $\mathrm{G}-\mathrm{III}-\mathrm{Br}(4.6 \mu \mathrm{M})$ with ethyl vinyl ether (EVE) in toluene at $5{ }^{\circ} \mathrm{C}$ have been re-visited. There is a rapid equilibrium between the bispyridyl form of $\mathrm{G}-\mathrm{II}-\mathrm{Br}, 1$, and its monopyridyl form, 2 $(K \approx 0.001 \mathrm{M})$. The empirical rate constants $\left(k_{\mathrm{obs}}\right)$ for the reaction with $E V E$, determined UV-vis spectrophotometrically under optimised anaerobic stopped-flow conditions, are found by testing the quality of fit of a series of steady-state approximations. The kinetics do not correlate with solely dissociative or associative pathways, but do correlate with a mechanism where these pathways converge at an alkene complex primed to undergo metathesis. In the presence of traces of air there is a marked increased in the rate of decay of G-III-Br due to competing oxidation to yield benzaldehyde; a process that appears to be very efficiently catalysed by trace metal contaminants. The apparent acceleration of the initiation process may account for the rates determined herein being over an order of magnitude lower than previously estimated.

\section{Introduction}

\section{Initiation in metathesis}

Over the last two decades, ruthenium alkylidene catalysed alkene metathesis ${ }^{1}$ has become ubiquitous, with applications at scales ranging from $\mu \mathrm{g}^{2}$ to $\mathrm{kg} .^{3}$ Much of this has been the direct consequence of a sustained period of development of a series of robust and well-characterised Ru-complexes, ${ }^{1}$ with three general mechanistic events ${ }^{4}$ identified as initiation, ${ }^{5,6}$ turnover, and decomposition. ${ }^{7}$ Initiation and turnover are mechanistically-related, and comprise, inter alia, alkene coordination, mutual interconversion of the alkylidene-alkene pair via a ruthenacyclobutane, then dissociation or displacement of the newly formed alkene. The co-product from initiation, most frequently styrene or a derivative, ${ }^{8}$ is non-innocent, able to undergo competitive metathetic reincorporation at ruthenium to regenerate the benzylidene complex. ${ }^{9}$ 
Exquisite detail regarding catalyst turnover has been elucidated using techniques such as heteronuclear NMR magnetisation transfer, ${ }^{\mathbf{1 0}}$ dynamic nuclear polarisation labelling, ${ }^{11}$ and single-molecule fluorescence. ${ }^{12}$ However, much of the work in the primary phases of the development of new ruthenium precatalysts, and the investigation of their relative efficiency and activity, focussed on the initiation event. ${ }^{\mathbf{1 , 4}, 5}$ A now classic example of such initiation studies is the series of experiments reported by Grubbs, Sanford and Love et al. in which a large excess of ethyl vinyl ether (EVE) was used to generate styrene and a stable Fischertype alkylidene product $[\mathrm{Ru}]=\mathrm{CHOEt}$, in an apparently irreversible manner. ${ }^{13}$ Using this approach, the kinetics of initiation, of both G-I and G-II, were shown to proceed via pathway I (Fig. 1). ${ }^{\mathbf{1 3}, \mathbf{1 4}}$

The initiation mechanisms for a wide variety of other Grubbs type catalysts have been studied, at varying levels of detail, both computationally ${ }^{15}$ and experimentally. ${ }^{5}$ Most salient to the results presented herein are the in-depth investigations by Plenio $^{16}$ and Percy ${ }^{17}$ of the initiation of the HG-II catalyst ${ }^{18}$ and analogues. ${ }^{16}$ The kinetic data were analysed either by the addition of a simple linear correction term, first order in alkene, to the standard hyperbolic fitting function for dissociative pathway I, or by the assumption of a second-order process. ${ }^{17}$ The kinetics ${ }^{16}$ and activation parameters ${ }^{17}$ were indicative that an associative 'interchange' (pathway IIa, Fig. 1) can also contribute to initiation..$^{\mathbf{1 6} 17}$

\section{The Grubbs Third Generation Catalysts (G-III-X)}

In 2001, Grubbs and Love reported the G-III-X (X $=\mathrm{H}, \mathrm{Cl}, \mathrm{Br})$ catalysts, which undergo exceptionally fast initiation. ${ }^{19}$ In preliminary studies using UV-vis
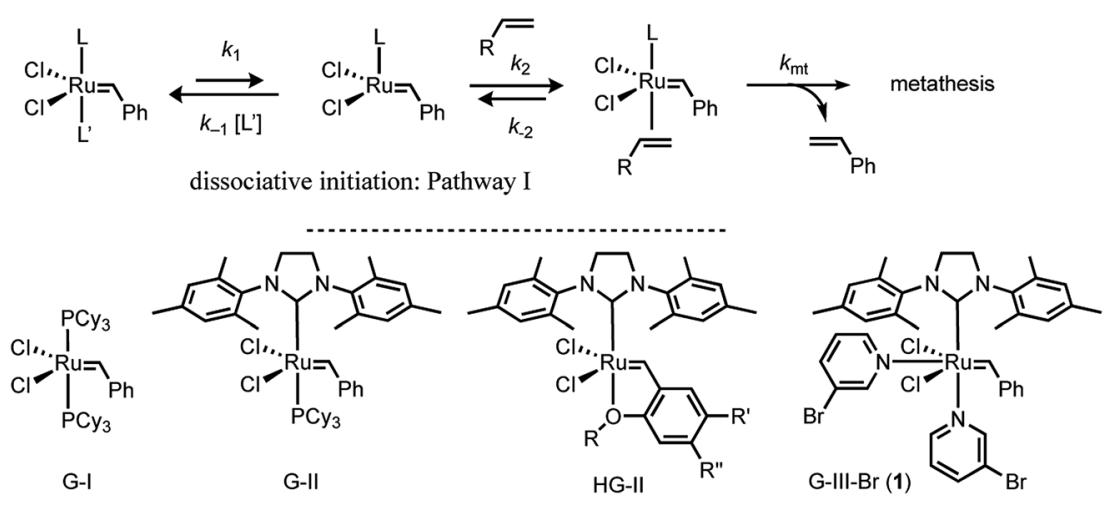

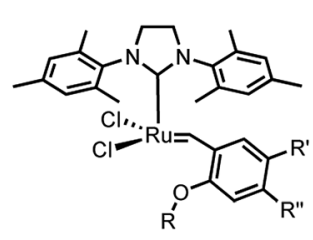

Pathway I HG-II

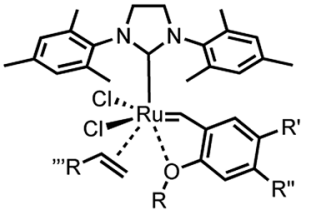

'interchange' Pathway IIa

HG-II

Fig. 1 Generic dissociative (pathway I) initiation mechanism for ruthenium-alkylidene complexes G-I and G-II and additional associative-interchange (pathway Ila) for HG-II. 
spectrophotometry, the lower-limit for the dissociative initiation $\left(k_{1}\right)$ of the fastest catalyst, G-III-Br, was estimated to be $>4 \mathrm{~s}^{-1}$; more than six orders of magnitude faster than G-II, ${ }^{13,14}$ and three orders of magnitude faster than HG-II. ${ }^{18}$ The readily-synthesised third generation system G-III-Br (1) remains one of the fastestinitiating commercially available ruthenium alkylidene metathesis catalysts. ${ }^{1}$

In 2013 Trzaskowski and Grela reported a computational analysis of the possible metathesis initiation pathways of the G-III-X pre-catalysts $(\mathrm{X}=\mathrm{Br}, \mathrm{H})$ by ethene and but-1-ene, Fig. $2 .^{20}$ A number of important conclusions were drawn. Firstly, the dissociation of a pyridine ligand from G-III-X (1 M standard state) was predicted to be only slightly endergonic, leading to $K_{0}$ values in the range of $10^{-3}$ to $10^{-1} \mathrm{M}$. Secondly, in complex 2 , the remaining pyridine ligand occupies a site that is intermediate between the cis and trans locations of the two pyridyl ligands in the precursor G-III-Br, 1. Thirdly, the dissociation $\left(k_{1}\right)$ of the remaining pyridine ligand from 2 , to generate the $14 \mathrm{e}^{-}$complex 3 , is also predicted to be only mildly endergonic, with a transition state barrier of between 10.1-13.6 $\mathrm{kcal} \mathrm{mol}^{-1}$; subsequent alkene coordination $\left(k_{2}\right.$, to generate complex 4, Fig. 2) has a low barrier $\left(<4 \mathrm{kcal} \mathrm{mol}^{-1}\right)$ and is mildly exergonic. Finally, a pathway involving association of the alkene with monopyridyl complex 2 to generate a discrete $18 \mathrm{e}^{-}$intermediate (analogous complex to 6, Fig. 2), en route to $16 \mathrm{e}^{-}$alkene complex 4 (stepwise associative, type IIb, $k_{4}$ ) was computed to have a similar barrier (at standard state) to dissociation (pathway I, $k_{1}$ ) and a lower barrier than the direct formation of 3 via interchange (IIa, $k_{3}$ ) at the $16 \mathrm{e}^{-}$intermediate 2 , Fig. 2. Thus overall, it was concluded, from DFT energetics, that dissociative (pathway

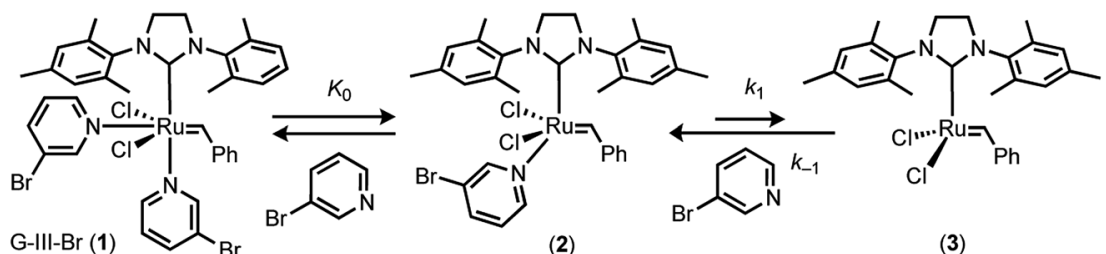

(2)

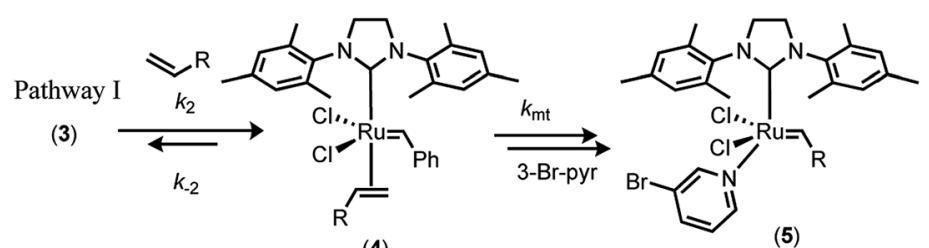

(4)

(5)

Interchange pathway IIa $(2 \rightarrow 4)$

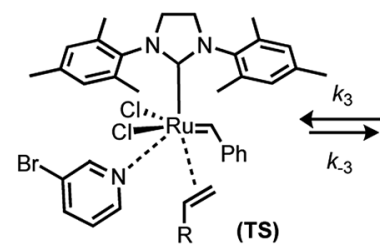

Associative pathway IIb $(2 \rightarrow 6 \rightarrow 4)$

(2)

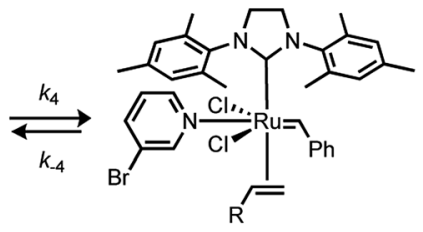

(6)

Fig. 2 Trzaskowski and Grela's computational analysis ${ }^{20}$ of mechanisms for initiation of the third-generation catalyst $\mathrm{G}-\mathrm{III}-\mathrm{Br}$ with ethene $(\mathrm{R}=\mathrm{H})$ and butene $(\mathrm{R}=\mathrm{Et})$. 
I) and associative-dissociative (pathways IIa, b) processes are all feasible mechanisms to initiation, and simultaneously that they are the rate-limiting steps for the overall initiation process. ${ }^{20}$

In 2017, Guironnet reported detailed NMR studies of the solution phase speciation of the G-III-X complexes $(\mathrm{X}=\mathrm{H}, \mathrm{Br})$ and the kinetics of polymerisation of $\mathrm{N}$ hexyl-exo-norbornene-5,6-dicarboximide. ${ }^{21}$ Titrations of G-III-X $(\mathrm{X}=\mathrm{H}, \mathrm{Br})$ in $\mathrm{CH}_{2} \mathrm{Cl}_{2}$ with pyridine, monitored by ${ }^{1} \mathrm{H} \mathrm{NMR}$, established that at room temperature, $K_{0}=0.4-0.5 \mathrm{M}$ for G-III-H, and $2.4 \mathrm{M}$ for G-III-Br $(1),{ }^{21}$ values broadly consistent with the calculations of Trzaskowski and Grela. ${ }^{20}$ Guironnet demonstrated that this has significant phenomenological impact on the metathesis kinetics, notably in livingpolymerisation, since the net effect of using G-III-Br 1 is that the pre-dissociated 3Br-pyridine (L), present at a concentration identical to the active species, 2, acts as an inhibitor to initiation and then turnover. Grubbs later reported on the copolymerization kinetics of 23 different norbornenyl comonomers with $\omega$-norbornenyl macromonomer, catalysed by G-III- $\mathrm{H}$, for which the ${ }^{1} \mathrm{H}$ NMR pyridine titrations of G-III-H in $\mathrm{CH}_{2} \mathrm{Cl}_{2}$ yielded $K_{0}=0.25 \mathrm{M}$, at room temperature. ${ }^{22}$ The polymerization kinetics were analysed on the basis of the dissociative mechanism (pathway I) in which a monoligated intermediate [(IMes)Ru( $=\mathrm{CH}$ poly $\left.) \mathrm{Cl}_{2}(3-\mathrm{H}-\mathrm{pyr})_{1}\right]$ (poly $=$ living polymer) is the dominant resting state in a pre-saturation regime $\left(k_{2}\right.$ $\left.\ll k_{-1}\right)$. However, it was noted that the associative pathway II, proceeding in a stepwise (IIb) or concerted (IIa) ${ }^{20}$ manner, could not be conclusively ruled out. ${ }^{21}$

Herein, we revisit the reaction of G-III-Br (1) with ethyl vinyl ether (EVE, 'E') in toluene at $5{ }^{\circ} \mathrm{C}$, Fig. $3,{ }^{19}$ to test whether the dissociative (I) and associative (II) pathways explored computationally by Trzaskowski and Grela, ${ }^{20}$ Fig. 2, can be distinguished experimentally. ${ }^{13,14,19}$
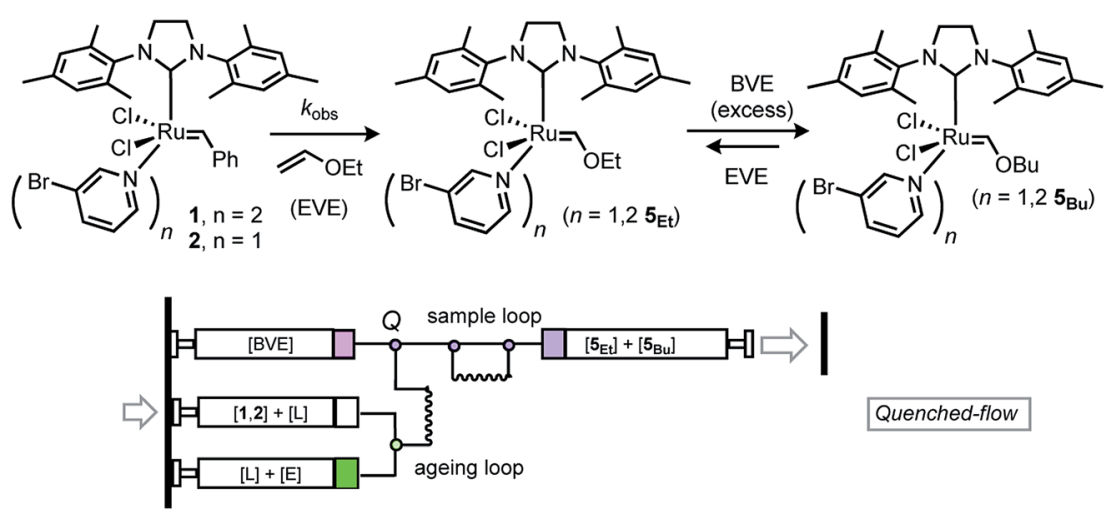

Quenched-flow

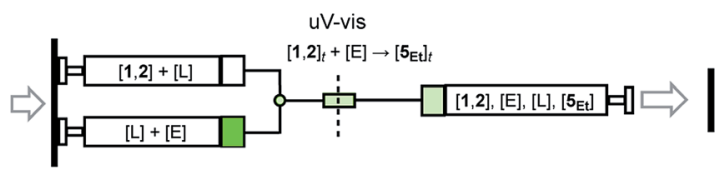

Stopped-flow

Fig. 3 Kinetics of initiation ( $k_{\text {obs. }}$ ) of $\mathrm{G}-\mathrm{III}-\mathrm{Br}(1,2+\mathrm{L})$ with excess ethyl vinyl ether (E, EVE), and schematics of mixing of reactants via rapid quenched-flow (RQF, with excess butyl vinyl ether (BVE) quenching agent, Q) and stopped-flow (SF-UV-vis). $\mathrm{PPh}_{3}$ and $\mathrm{PCy}_{3}$ proved inefficient as quenching reagents. 


\section{Results and discussion}

Initial tests confirmed that the alkylidene $\mathrm{Ru}=\mathrm{CH}$ signal in the ${ }^{1} \mathrm{H}$ NMR spectra of G-III-Br versus Fischer carbene $\mathbf{5}_{\mathrm{Et}}$ was well resolved in toluene (19.4 $\mathrm{ppm}$ versus $13.8 \mathrm{ppm}$, respectively), and that addition of $\geq 1.1$ equivalents of EVE to 1 and 2 led to $>99 \%$ conversion to $\mathbf{5}_{\mathrm{Et}}$. We began by exploring automated rapid quenched-flow (RQF, Fig. 3) using butyl vinyl ether, BVE, in large excess (100 equiv.) as a quenching agent $(Q)$. However, the control experiments showed that, somewhat surprisingly, $\mathbf{5}_{\mathrm{Et}} / \mathbf{5}_{\mathrm{Bu}}$ undergo interconversion, leading to the erosion of the kinetic trapping ratio of $\mathbf{5}_{\mathrm{Et}} / \mathbf{5}_{\mathrm{Bu}}$, over a period of minutes, even after adding $\mathrm{PPh}_{3}$ as a stabiliser.

\section{Stopped-flow UV-vis analysis}

Given the above complications, we turned to the analysis of the reactions by in situ UVvis spectrophotometric analysis. ${ }^{19}$ Using a stopped-flow system, Fig. 3, we monitored the signal centred at $354 \mathrm{~nm}$, assumed to arise from metal to benzylidene $\pi^{*}$ charge transfer analogous to that with $\mathrm{H}-\mathrm{G}-\mathrm{II},{ }^{\mathbf{1 6}}$ and absent in the initiation product $([\mathrm{Ru}]=$ CHOEt), which lacks the conjugated phenyl group. Whilst this in situ method leads to a substantial increase in data-density compared to e.g. RQF, the low concentrations of $[\mathrm{Ru}]_{\text {tot }}$ (approx. $50 \mu \mathrm{M}$ ) required for the UV-vis analysis initially resulted in some variability in rates between runs. This was eventually identified as arising from trace diffusion of air into reagent delivery lines, the solvent-purification system, and into sample reservoirs in the stopped-flow apparatus, which led to periods during which the first-order rate of quenching of the UV absorption at $348 \mathrm{~nm}$ increased sporadically between runs. The deliberate oxidation of G-III-Br in $\mathrm{d}_{8}$-toluene was monitored by ${ }^{1} \mathrm{H}$ NMR spectroscopy, which confirmed benzaldehyde generation by the oxidative cleavage of the benzylidene ligand, and thus the loss of the moiety responsible for the MLCT band; the complex mixture of ruthenium co-products was not further investigated. Subsequent tests confirmed that the aerobic oxidation reaction of dilute solutions of G-III-Br $(\mathbf{1}, \mathbf{2})$ in toluene solution, as monitored by the decay of the MLCT band at $354 \mathrm{~nm}$, is not photocatalysed (decay rates were unaffected by gating the UVvis irradiation) but is highly sensitive to the method of stock-solution preparation and its introduction into the stopped-flow system. The passage of the solution of G-III-Br through stainless-steel needles during sample preparation (sequential dilutions etc.) or during introduction to the apparatus induces very rapid oxidative decay rates, as compared to samples prepared and delivered solely through glass/PTFE systems. $\dagger^{\dagger}$ After extensive procedural refinement, the rates of decay of the MLCT band became stable and reproducible, within experimental error, between samples and runs, including attenuation to zero when no EVE was present, and thus attributable solely to metathesis initiation, rather than other competing processes.

\footnotetext{
$\dagger$ There was no impact from the use of glass-bodied syringes with PTFE-tipped plungers versus disposable plastic syringes or versus ground-glass barrel/plunger type syringes. The stainless-steel needles employed for the work are brazed or compression-bonded to a nickel-plated brass Luer-lock hub. The possibility that the 3-Br-pyridine ligand leaches traces of metal from the inside surface of the needle bore (e.g. $\mathrm{Ni}, \mathrm{Fe}, \mathrm{Mn}, \mathrm{Cr}, \mathrm{Mo}$ ) or Luer-lock hub (e.g. $\mathrm{Ni}, \mathrm{Cu}, \mathrm{Zn})$ is consistent with the observations; there was substantial variability, in terms of impact on the rate of oxidation, between nominally identical needles that were tested. We are currently investigating this phenomenon in more detail, including testing homogeneous solutions of the simple pyridyl complexes of a range of transition metals as catalysts for the aerobic oxidation of G-III-Br.
} 


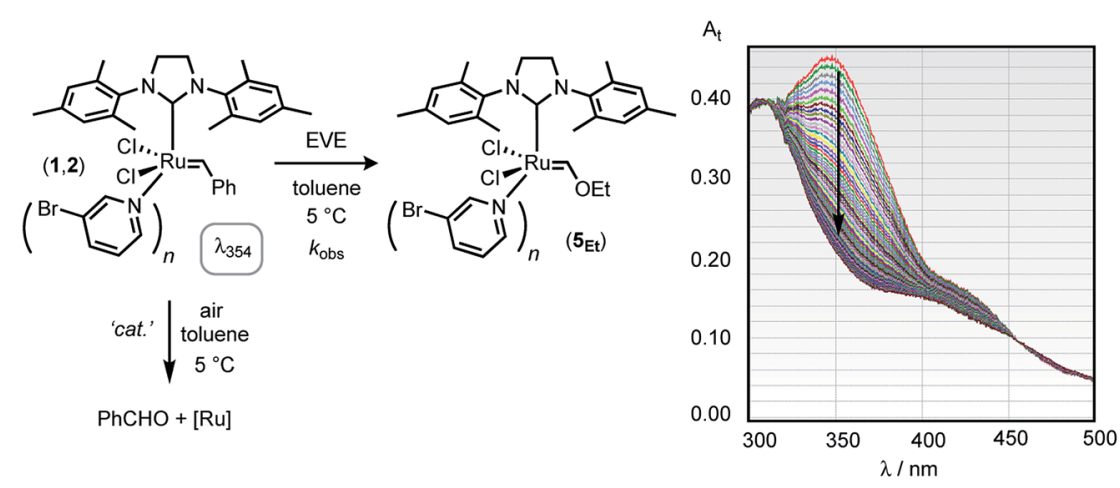

Fig. 4 General scheme for kinetics of the initiation ( $\left.k_{\mathrm{obs}}\right)$ of $\mathrm{G}-\mathrm{III}-\mathrm{Br}(1,2+\mathrm{L})$ with excess ethyl vinyl ether (E, EVE), and example series of 200 transient UV spectra, $\Delta t_{n, n+1}=40 \mathrm{~ms}$, obtained on mixing $1,2+\mathrm{L}\left([R]_{\text {tot }}=[L]_{\text {tot }}=0.046 \mathrm{mM}\right)$ with ethyl vinyl ether $\left([E]_{0}=0.83 \mathrm{M}\right)$.

\section{Kinetics of reaction of EVE with G-III-Br}

Using the stopped-flow method, the kinetic data were obtained from the temporal decay of the signal at $354 \mathrm{~nm}$, Fig. 4, obtained approximately $8 \mathrm{~ms}$

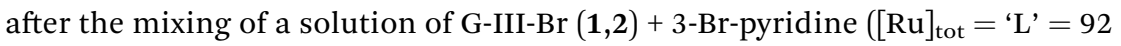
$\mu \mathrm{M}$ ) with solutions of ethyl vinyl ether ('E', $0.8 \mathrm{mM}$ to $1.66 \mathrm{M}$ ) in toluene at 5.0 $\pm 0.1{ }^{\circ} \mathrm{C}$. In further experiments, the solution of ethyl vinyl ether also contained 3-Br-pyridine (' $\mathrm{L}$ ', 0 to $4.2 \mathrm{mM}$ ). The empirical first order rate constants were obtained by non-linear regression of the exponential decay in absorbance, Fig. 5. First-order decays in the signal were obtained through $>6$ half-lives ( $>98 \%$ conversion) under all conditions tested. The empirical rate constants, $k_{\text {obs. }}$, Table 1 , were then determined by averaging a minimum of 10 experiments per concentration variation. For comparison, analogous experiments were conducted with the slower-initiating G-I catalyst system, at [EVE] concentrations ranging from 0.1 to $1.5 \mathrm{M}$, again in toluene, but at $21{ }^{\circ} \mathrm{C}$, Table 2 .
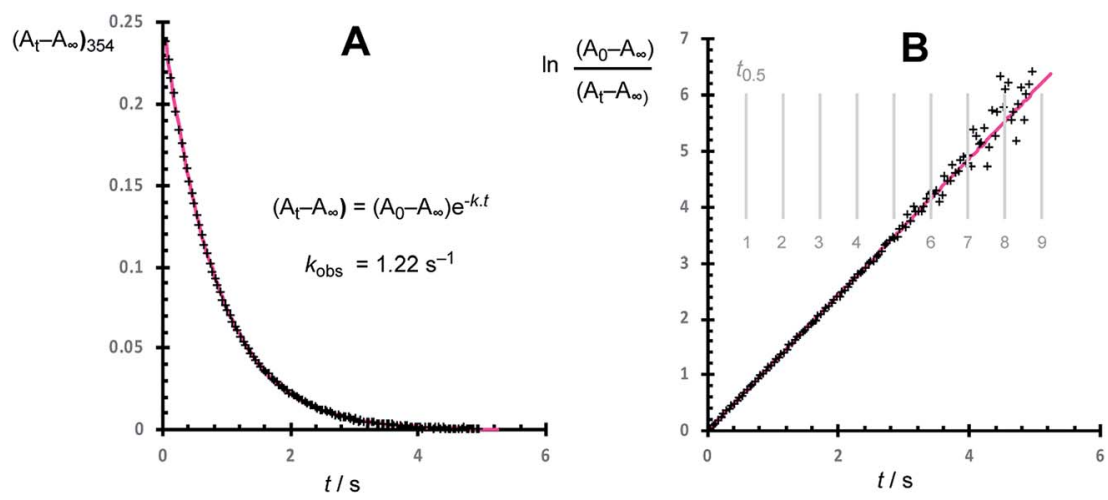

Fig. 5 (A) Example analysis of the kinetics of initiation ( $\left.k_{\text {obs. }}\right)$ of $\mathrm{G}-\mathrm{III}-\mathrm{Br}(1,2+\mathrm{L})$ with excess ethyl vinyl ether ( $E$, EVE) in toluene at $5^{\circ} \mathrm{C}$. (A) Exponential decay of the signal at $354 \mathrm{~nm}$, with nonlinear regression, $k=1.22 \mathrm{~s}^{-1}$. (B) First-order behaviour in decay of 1,2 with half-lives indicated. 
Table 1 First-order empirical rate constants $\left(k_{\text {obs }} / \mathrm{s}\right)$ for decay of 1,2 by reaction with ethyl vinyl ether in toluene at $5{ }^{\circ} \mathrm{C}$, monitored using stopped-flow UV-vis

\begin{tabular}{|c|c|c|c|}
\hline Entry & {$[\mathrm{EVE}]_{0} / \mathrm{M}$} & {$[\mathrm{L}]_{\text {added }} / \mathrm{mM}$} & $k_{\text {obs. }}{ }^{a} / \mathrm{s}$ \\
\hline 1 & 0.063 & 0.000 & $0.29(0.03)$ \\
\hline 2 & 0.310 & 0.000 & $0.57(0.03)$ \\
\hline 3 & 0.520 & 0.000 & $0.90(0.05)$ \\
\hline 4 & 0.630 & 0.000 & $1.06(0.05)$ \\
\hline 5 & 0.690 & 0.000 & $1.00(0.02)$ \\
\hline 6 & 0.830 & 0.000 & $1.26(0.05)$ \\
\hline 7 & 0.063 & 0.250 & $0.18(0.02)$ \\
\hline 8 & 0.310 & 0.250 & $0.31(0.02)$ \\
\hline 9 & 0.630 & 0.250 & $0.50(0.03)$ \\
\hline 10 & 0.830 & 0.250 & $0.63(0.01)$ \\
\hline 11 & 0.063 & 0.446 & $0.15(0.01)$ \\
\hline 12 & 0.310 & 0.446 & $0.24(0.01)$ \\
\hline 13 & 0.630 & 0.446 & $0.36(0.02)$ \\
\hline 14 & 0.830 & 0.446 & $0.44(0.007)$ \\
\hline 15 & 0.063 & 1.046 & $0.10(0.01)$ \\
\hline 16 & 0.310 & 1.046 & $0.14(0.01)$ \\
\hline 17 & 0.630 & 1.046 & $0.22(0.01)$ \\
\hline 18 & 0.830 & 1.046 & $0.24(0.01)$ \\
\hline 19 & 0.063 & 2.050 & $0.06(0.003)$ \\
\hline 20 & 0.310 & 2.050 & $0.09(0.007)$ \\
\hline 21 & 0.630 & 2.050 & $0.12(0.01)$ \\
\hline 22 & 0.830 & 2.050 & $0.15(0.01)$ \\
\hline 23 & 0.0004 & 0.000 & $0.06(0.002)$ \\
\hline 24 & 0.0020 & 0.000 & $0.14(0.01)$ \\
\hline 25 & 0.0040 & 0.000 & $0.21(0.02)$ \\
\hline 26 & 0.0063 & 0.000 & $0.22(0.03)$ \\
\hline 27 & 0.0340 & 0.000 & $0.29(0.03)$ \\
\hline $28^{b}$ & 0.063 & 0.000 & $0.35(0.05)$ \\
\hline
\end{tabular}

Table 2 First-order empirical rate constants $\left(k_{\text {obs. }} / \mathrm{s}\right)$ for decay of G-I by reaction with ethyl vinyl ether in toluene at $21^{\circ} \mathrm{C}$, monitored using stopped-flow UV-vis

\begin{tabular}{llll}
\hline Entry & {$[\mathrm{EVE}]_{\mathrm{o}} / \mathrm{M}$} & {$[\mathrm{L}]_{\text {added }} / \mathrm{mM}$} & $k_{\text {obs. }}{ }^{a} / \mathrm{s}$ \\
\hline 1 & 0.146 & 0.000 & $0.0070(0.0002)$ \\
2 & 0.198 & 0.000 & $0.0079(0.0002)$ \\
3 & 0.245 & 0.000 & $0.0080(0.0002)$ \\
4 & 0.371 & 0.000 & $0.0097(0.0002)$ \\
5 & 0.493 & 0.000 & $0.0110(0.0001)$ \\
6 & 0.618 & 0.000 & $0.0110(0.0001)$ \\
7 & 0.668 & 0.000 & $0.0108(0.0001)$ \\
8 & 0.718 & 0.000 & $0.0113(0.0001)$ \\
9 & 0.912 & 0.000 & $0.0119(0.0001)$ \\
10 & 1.500 & 0.000 & $0.0123(0.0002)$
\end{tabular}

${ }^{a} k_{\text {obs. }}$ is an average of $\geq 10$ experiments, obtained by non-linear regression of growth of the signal at $484 \mathrm{~nm}$. Standard deviation $(1 \sigma)$ in parenthesis. $[\mathrm{Ru}]_{\text {tot }}=0.046 \mathrm{mM}$. Concentrations are those after mixing at $21 \pm 0.1^{\circ} \mathrm{C} .{ }^{b}$ No additional ligand was added. 
As anticipated, ${ }^{\mathbf{1 3} \mathbf{1 4}}$ the data obtained for the G-I system (Table 2) gave a simple correlation with [EVE], as analysed graphically, Fig. 6A, by the application of the standard steady-state rate equation for pathway I, yielding an initiation rate $\left(k_{1}\right)$ of $0.014 \pm 0.002 \mathrm{~s}^{-1}$ at $21{ }^{\circ} \mathrm{C}$, in agreement with the value reported by Sanford, Ulman and Grubbs $\left(0.016 \mathrm{~s}^{-1}\right) .^{13}$

In contrast to G-I, the application of the analogous standard steady-state rate equation for pathway I, ${ }^{14-16}$ for the data obtained with G-III-Br (Table 1), did not give a simple linear correlation, Fig. 6B, which was indicative that dissociation $\left(k_{1}\right)$ does not dominate initiation across the range of concentrations tested. The reactions conducted without added 3-Br-pyridine (L) and with ethyl vinyl ether (E) concentrations in the range 0.06 to $0.83 \mathrm{M}$ (Table 1, entries 1-6) gave rates that were linearly dependent on [E]; but had a substantial non-zero $y$-axis intercept: $k_{\text {obs. }}=a[\mathrm{E}]+b$; Table 3 , entry 1 . The kinetics deviated strongly from the

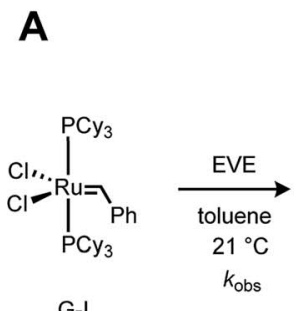

G-I
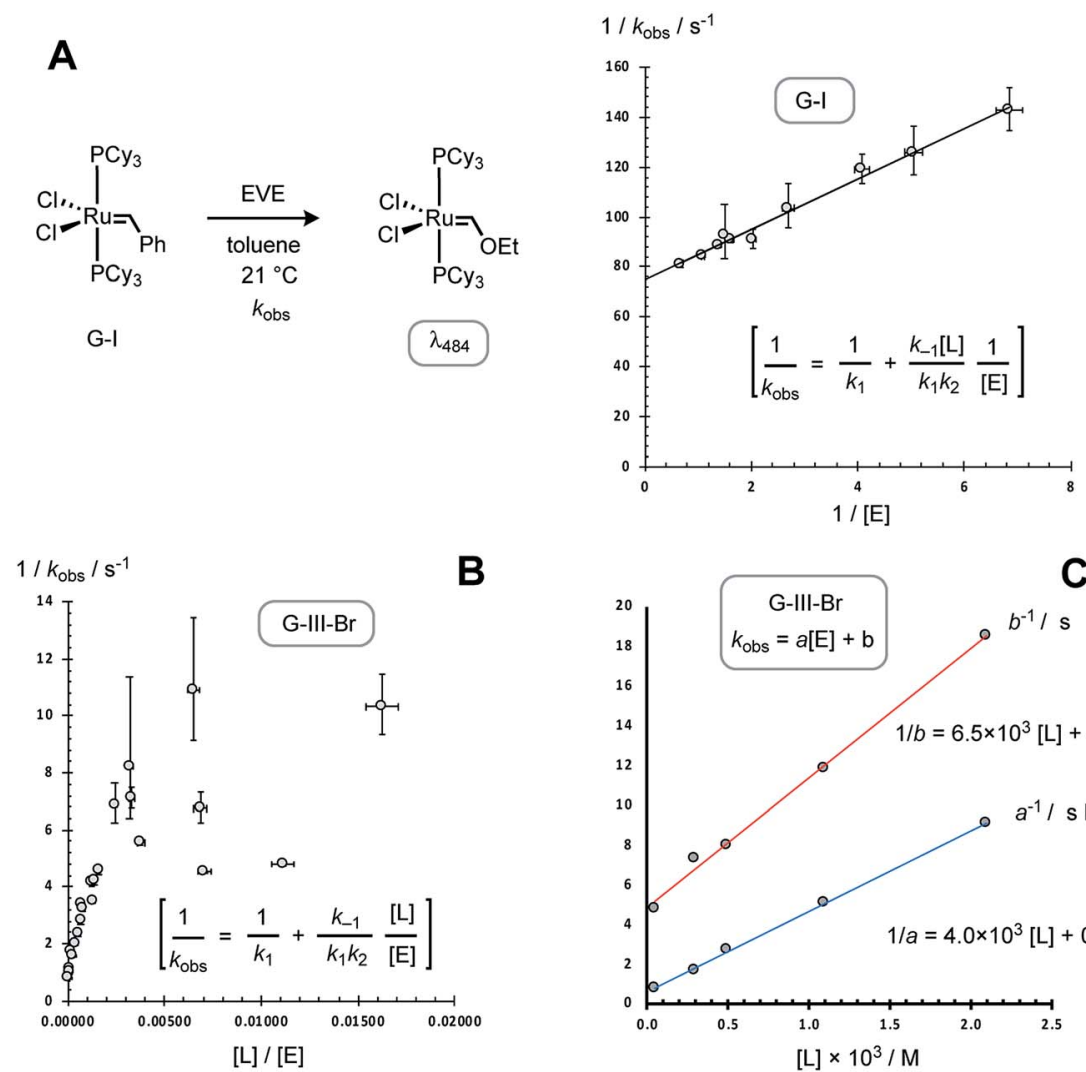

B

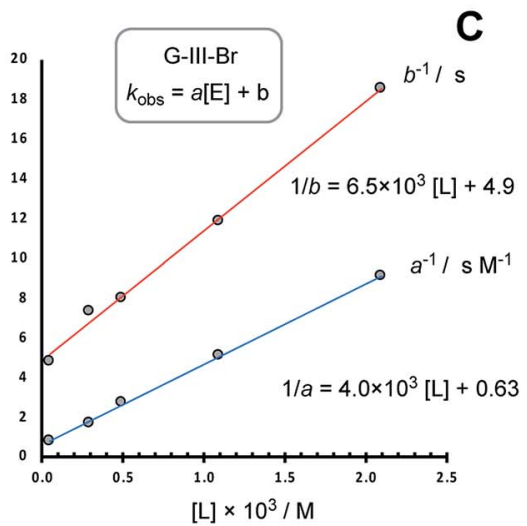

Fig. 6 Preliminary analyses of rate-data from Tables 1 and 2, employing the equation for initiation solely via dissociative pathway I (see Fig. 2 for rate constant definitions). (A) G-I initiation at $21^{\circ} \mathrm{C}$; correlation: $1 / k_{\text {obs }}=10.5 /[\mathrm{E}]+74$, leads to $k_{1}=0.014 \mathrm{~s}^{-1}$. Error bars are propagated from $\pm 1 \sigma$ in $k_{\mathrm{obs}}$ and $\pm 2.5 \%$ in [E]. (B) G-III-Br initiation at $5{ }^{\circ} \mathrm{C}$; scattered correlation. Table 1 entries 19 and 23 omitted for clarity. [L] calculated from $K_{0}=0.001 \mathrm{M}$. Error bars are propagated from $\pm 1 \sigma$ in $k_{\mathrm{obs}}$ and $\pm 2.5 \%$ in both [L] and [E]. (C) Correlation of empirical coefficients for $k_{\mathrm{obs}}=a[\mathrm{E}]+b$, Table 3 , with $[\mathrm{L}]_{\mathrm{tot}}$, leading to $k_{\mathrm{obs}} / \mathrm{s}=([\mathrm{E}] /(4.0 \times$ $\left.\left.10^{3}[\mathrm{~L}]+0.63\right)\right)+1 /\left(6.5 \times 10^{3}[\mathrm{~L}]+4.9\right)$, when $0.83 \geq[\mathrm{E}] \geq 0.06 \mathrm{M}$ and $[\mathrm{L}]$, calculated using $K_{0}=0.001 \mathrm{M}$, is in the range 0.05 to $2.1 \mathrm{mM}$. 
Table 3 Empirical coefficients, $a$ and $b$, at various ligand ([L]) concentrations, for the dependence of first-order rate empirical rate constant $k_{\mathrm{obs}}=a[\mathrm{E}]+b$, for the reaction of $\mathrm{G}-\mathrm{III}-\mathrm{Br}$ with ethyl vinyl ether $(\mathrm{E})$ in the range $[\mathrm{E}]_{0}=0.06$ to $0.83 \mathrm{M}$, toluene, $5^{\circ} \mathrm{C}$

\begin{tabular}{lccc}
\hline Entry & {$[\mathrm{L}]_{\text {tot }}{ }^{a} / \mathrm{mM}$} & $a / \mathrm{M}^{-1} \mathrm{~s}^{-1}$ & $b / \mathrm{s}^{-1}$ \\
\hline 1 & 0.046 & 1.26 & 0.21 \\
2 & 0.296 & 0.59 & 0.14 \\
3 & 0.492 & 0.36 & 0.12 \\
4 & 1.092 & 0.20 & 0.08 \\
10 & 2.096 & 0.11 & 0.05 \\
${ }^{a}[\mathrm{Ru}]_{\text {tot }}=0.046 \mathrm{mM} .[\mathrm{L}]$ includes endogenous 3-Br-pyridine, calculated using $K_{0}=0.001 \mathrm{M}$. \\
Reactions in toluene at $5 \pm 0.1^{\circ} \mathrm{C}$.
\end{tabular}

relationship $k_{\mathrm{obs} .}=a[\mathrm{E}]+b$ when $[\mathrm{E}]_{0} \leq 4 \mathrm{mM}$ (Table 1, entries 23-27); see below for further discussion and global kinetic analysis. The inclusion of exogenous ligand $([\mathrm{L}]=0.3-2 \mathrm{mM}$, Table 1 , entries $7-22)$ resulted in progressive reduction in the empirical coefficients $a$ and $b$, Table 3, entries 2-5. Graphical analysis showed that both $1 / a$ and $1 / b$ have an approximately linear dependence on [L], Fig. 6C, indicative of inhibition by added ligand L, via at least two pathways.

\section{Ligand dissociation from G-III-Br}

Guironnet ${ }^{21}$ and Grubbs, ${ }^{22}$ have independently determined $K_{0}$ for G-III-H and G-III$\mathrm{Br}$ in $\mathrm{CH}_{2} \mathrm{Cl}_{2}$. The values from van't Hoff analysis of G-III-H, ${ }^{21}$ together with the $\left(K_{0}^{\mathrm{Br}} /\right.$ $K_{0}{ }^{\mathrm{H}}$ ) ratio of 4.8, might then suggest that for the reactions reported herein there will be $>99 \%$ dissociation of 1 to 2 . However, this requires a negligible impact of solvent $\left(\mathrm{CH}_{2} \mathrm{Cl}_{2}\right.$ versus toluene) on the equilibrium. G-III-Br was thus analysed by ${ }^{1} \mathrm{H} \mathrm{NMR}$ spectroscopy in $\mathrm{d}_{8}$-toluene at $5{ }^{\circ} \mathrm{C}$, combining data from a series of additions of 3bromopyridine ligand to G-III-Br, and a series of dilutions of G-III-Br without added ligand. The linear regression of the time-average population-weighted chemical shift of the benzylidene $\mathrm{CH}$ signal (19.3-19.5 ppm) against that calculated using a standard quadratic equation for the equilibrium of 1 with 2 , with $K_{0}, \delta_{1}$ and $\delta_{2}$ as variables, Fig. 7, established that ligand dissociation from $\mathbf{1}$ is considerably less favourable in toluene at $5{ }^{\circ} \mathrm{C}$, compared to $\mathrm{CH}_{2} \mathrm{Cl}_{2}$ at room temperature.

Given the sensitivity of the equilibrium to solvent, the initiation rate of G-III-Br conducted at high concentrations of ethyl vinyl ether, [E], could conceivably be affected by the change in medium from pure toluene to mixtures of toluene and ethyl vinyl ether. A control experiment in which the reaction was conducted in toluene containing $0.063 \mathrm{M}$ ethyl vinyl ether and $0.767 \mathrm{M} \mathrm{Et}_{2} \mathrm{O}$, as a nonmetathetic surrogate, gave a similar empirical rate constant to that conducted with just the ethyl vinyl ether in toluene (Table 1, entries 1 and 28), indicative that the changing reaction medium does not contribute significantly to the observed increase in empirical coefficient $a$ as [E] is increased.

\section{Steady-state analyses of dissociative and associative initiation mechanisms for G-III-Br}

A dissociative mechanism beginning from 2 , and proceeding via $\mathbf{3}$ and $\mathbf{4}$, to irreversibly generate 5 will result in the rate of metathesis rising as the ethyl vinyl ether concentration, [E], is raised, but in a decreasingly proportionate manner, 


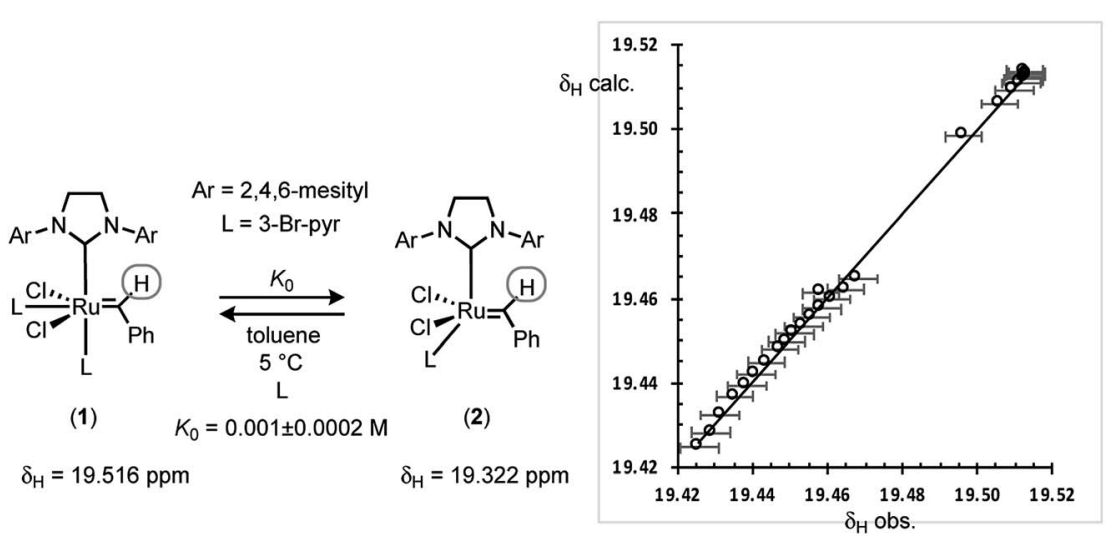

Fig. $7 \quad{ }^{1} \mathrm{H}$ NMR analysis of the chemical shift of the benzylidene- $\mathrm{CH}$ in $\mathrm{G}-\mathrm{III}-\mathrm{Br}$ in $\mathrm{d}_{8}$ toluene at $5{ }^{\circ} \mathrm{C}$ as a function of concentration of $1\left([R u]_{\text {tot }}=2.1\right.$ to $\left.9.7 \mathrm{mM}\right)$ and $3-\mathrm{Br}-$ pyridine $\left(8.9-89 \mathrm{mM}\right.$ at $\left.[R u]_{\text {tot }}=8.33 \mathrm{mM}\right)$. Linear regression of the observed $(x$-axis) timeaverage population-weighted $(1+2)$ chemical shift of the benzylidene- $\mathrm{CH}$ against that calculated ( $y$-axis) when $K_{0}=0.001 \mathrm{M}, \delta_{1}=19.516 \mathrm{ppm}$ and $\delta_{2}=19.322 \mathrm{ppm}$, referenced against residual $d_{7}$-toluene $\mathrm{CHD}_{2}=2.128 \mathrm{ppm}$. Correlation: $\delta_{\text {calc. }}=0.9981\left(\delta_{\text {obs }}\right)+0.0361$; RMSE, \pm 0.00005 , yielding $K_{0}=0.001 \pm 0.0002 \mathrm{M}$. Error bars in $\delta_{\text {obs }}$ are at $0.005 \mathrm{ppm}$.
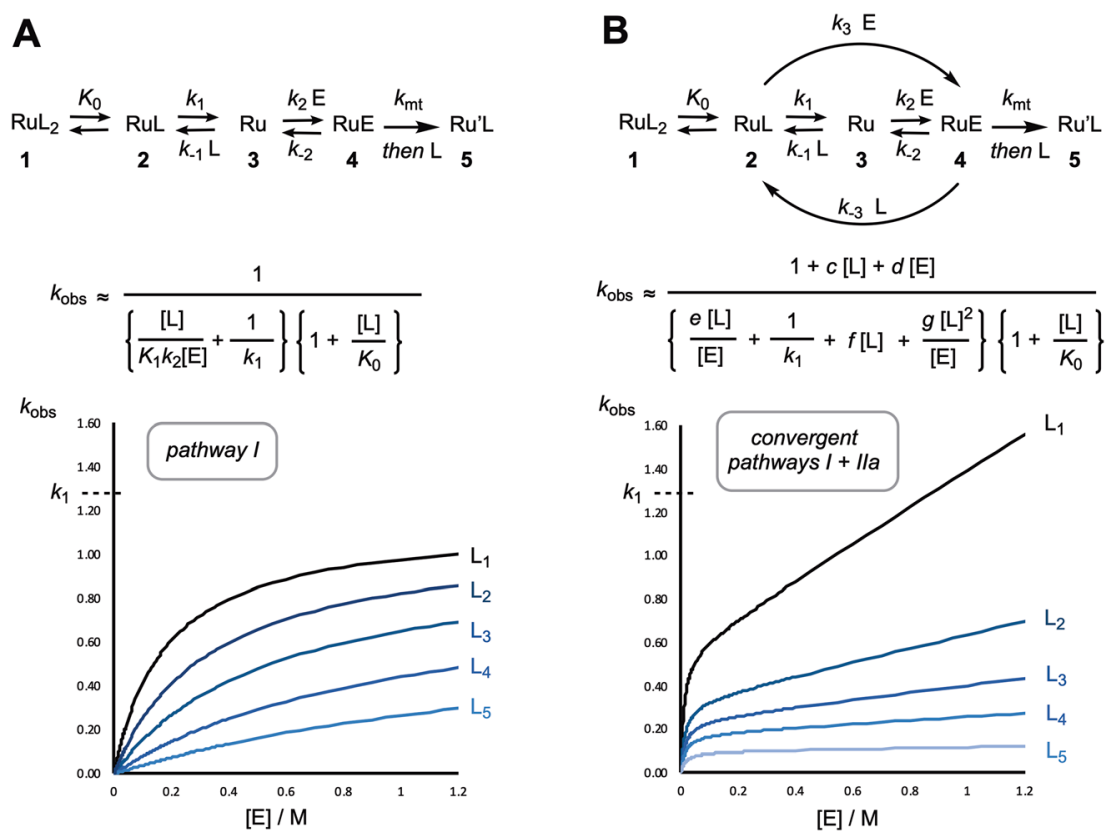

Fig. 8 Generic kinetic profiles (arbitrary values; $y$-axis: $k_{\text {obs. }} x$-axis: alkene concentration (E)) for initiation of a $\mathrm{RuL}_{2}$ complex, at various exogenous ligand concentrations $\left(\mathrm{L}_{1-5}\right)$ via a solely dissociative mechanism (pathway I, A) versus a mechanism in which dissociative and associative processes converge at alkene complex 4 (pathways I + Ila; B), where $c=$ $k_{3} / K_{1} k_{2}, d=k_{3} / k_{1}, e=\left(1 / k_{1} k_{2} k_{\mathrm{mt}}+1 / K_{1} k_{2}\right), f=k_{-3} / k_{1} k_{\mathrm{mt}}, g=k_{-3} / K_{1} k_{2} k_{\mathrm{mt}}$. The fast preequilibrium $K_{0}$ determines the mole fraction [2] for the steady-state approximation. 
eventually reaching an asymptote when $k_{2} k_{\mathrm{m}}[\mathrm{E}] \gg k_{-1}[\mathrm{~L}]\left(k_{-2}+k_{\mathrm{m}}\right)$, such that the overall rate of conversion of 2 to 5 becomes independent of [E] and is dictated solely by the rate of ligand (L) dissociation $\left(k_{1}\right)$; i.e. saturation kinetics. Based on the pyridine dissociation constant $\left(K_{0}\right)$ determined herein (Fig. 7$)$ the speciation of the G-III-Br complex 1 in solution under the conditions employed for stoppedflow UV-vis will vary from being dominated by monopyridyl complex 2 (96\%), when no exogenous 3-bromopyridine ligand (L) is present, through to predominantly the bispyridyl complex $1(66 \%)$ when $\mathrm{L}=2 \mathrm{mM}$. Thus, as the free ligand concentration, [L], is raised (Table 1, entries 7-22) the rate of metathesis will be attenuated in both the pre-saturation phase and the saturation phase, as outlined schematically in Fig. 8A.

However, even with this adjustment for $K_{0}$, the single-pathway dissociative mechanism (pathway I) is inconsistent with the observed dependencies on [E] and $[\mathrm{L}]$, Table 1 . The augmentation of the steady-state rate equation for pathway I with an additional term that solely comprises a divergent associative pathway (IIa or IIb) ${ }^{16}$ gives a better, but still imperfect, correlation. However, non-linear regression of the predicted versus empirical rate constant $\left(k_{\text {obs. }}\right)$ as a function of [E] and [L] using a speciation-weighted $\left(K_{0}\right)$ steady-state rate equation in which 2 undergoes conversion to 5 via convergence of pathways I and II at intermediate 4 (Fig. 8B) gives a good overall correlation, Fig. 9A, including data at low concentrations of $[\mathrm{E}]$ and $[\mathrm{L}]$, where the dominant process is dissociative (pathway I), see inset to Fig. 9B. A key component in the steady-state analysis for the convergent mechanism I + II is that the dissociative pathway via $\mathbf{2} \rightarrow \mathbf{3} \rightarrow \mathbf{4}$
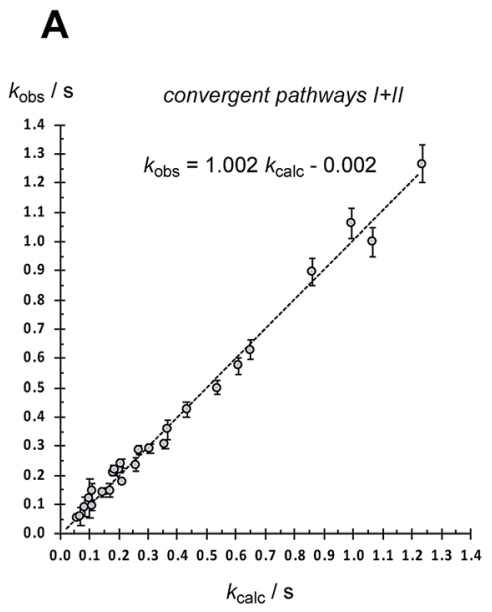

B

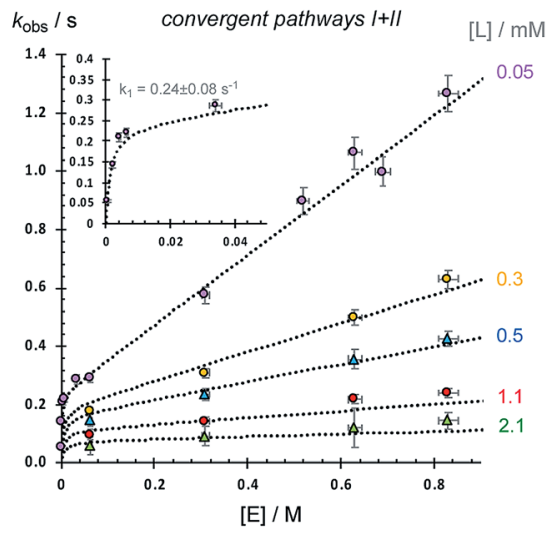

Fig. 9 Analysis using steady-state rate equation for convergent mechanism I + II (Fig. 8B) and data from Table 1. $k_{\text {calc. }}=x_{2}\left\{\left(1+7 \times 10^{3}[\mathrm{~L}]+6.1[\mathrm{E}]\right) /\left(1.2 \times 10^{2}[\mathrm{~L}] /[\mathrm{E}]+2.7 \times 10^{4}[\mathrm{~L}]+\right.\right.$ $\left.\left.5 \times 10^{5}[\mathrm{~L}]^{2} / \mathrm{E}+4.2\right)\right\}$; see Fig. $8 \mathrm{~B}$. (A) Correlation of $k_{\mathrm{obs}}$. with $k_{\text {calc. }} ; R^{2}=0.991$. Error bars for $k_{\text {obs }}$ are plotted at the greater of $5 \% /$ standard deviation $( \pm 2 \sigma)$ (B) Correlations of $k_{\text {obs }}$. with [E] (0.0004 to $0.83 \mathrm{M})$ at various free ligand $\mathrm{L}$ concentrations, as indicated. Error bars are plotted at the greater of $5 \% /$ standard deviation $( \pm 2 \sigma)$ for $k_{\text {obs., }}$ and $\pm 2.5 \%$ for [E]. Dotted lines $=k_{\text {calc }}$. Inset shows region where pathway (I) dominates. In the calculations, $x_{2}$ is the mole fraction $[2] /[\mathrm{Ru}]$ and $[\mathrm{L}]=[2]+[\mathrm{L}]_{\text {exog. }}$. Both are calculated using a standard quadratic equation for the equilibrium between 1 and 2, based on $K_{0}=0.001 \mathrm{M}$; see Fig. 11, eqn $(6-8)$. 
can be attenuated by the direct conversion of 4 to 2, (or 6), by [L], which phenomenologically appears to attenuate the saturation limit $\left(k_{1}=0.24( \pm 0.08)\right.$ $\mathrm{s}^{-1}$ in toluene at $5{ }^{\circ} \mathrm{C}$ ).

\section{Conclusions}

The kinetics of the nominally irreversible reaction of the third generation Grubbs catalyst G-III-Br with ethyl vinyl ether (E) in toluene at $5{ }^{\circ} \mathrm{C}$ have been revisited. ${ }^{19}$ The rapid equilibrium between the bispyridyl form of G-III-Br, 1, and the monopyridyl form, 2, was analysed by ${ }^{1} \mathrm{H}$ NMR titration/dilution, yielding $K_{0}=0.001 \mathrm{M}$. The empirical pseudo first-order rate constants ( $k_{\mathrm{obs}}$ ) for the kinetics of the reaction of G-III-Br $(46 \mu \mathrm{M})$ with ethyl vinyl ether (E), to generate $\mathbf{5}_{\mathrm{Et}}$, have been determined under anaerobic stopped-flow conditions by the UVvis spectrophotometric analysis of the decay of the MLCT band at $354 \mathrm{nM}$. Across the range of concentrations of the exogenous ligand, [L], explored, the speciation of G-III-Br ranges from $33 \%$ to $96 \% 2$, based on $K_{0}=0.001 \mathrm{M}$. There was no evidence for any significant accumulation of intermediates, decays in $[\mathbf{1}, \mathbf{2}]$ were clean first-order, and the isosbestic points in the UV-vis spectra were retained.

The empirical rate constants obtained $\left(k_{\text {obs. }}\right)$ were analysed in detail by testing the quality of fit of a series of steady-state approximations. The kinetics correlate with a mechanism where the dissociative and associative pathways converge at the pyridyl-free complex $\mathbf{4}$ in which the alkene is bound and primed to undergo metathesis with the benzylidene. The analysis (Fig. 8 and 9) requires that complex 4 has a lifetime, and thus $k_{\mathrm{mt}}<\left(k_{-3}[\mathrm{~L}]+k_{-2}\right)$, i.e. 4 , can revert to both 2 and 3 , or progress to 5 .

The previous analyses of the initiation kinetics for the Grubbs catalysts, and related species, have predominantly, but not exclusively, ${ }^{16,17}$ been by the application of a steady-state dissociative mechanism (pathway I). ${ }^{13-22}$ However, our study strongly supports the conclusions of Trzaskowski and Grela ${ }^{20}$ that both the associative and dissociative pathways are kinetically relevant in the initiation, and thus also propagation, of G-III systems. Indeed, the higher the alkene concentration, the more dominant the associative pathway, Fig. 10. In principle, saturation kinetics can be achieved for G-III-Br at high alkene [E] concentrations and low ligand $[\mathrm{L}]$ concentrations, where the steady-state approximation for growth of metathesis product 5 eventually reduces to $k_{\mathrm{obs}} \approx k_{\mathrm{mt}}$ (IIa and IIb) or $k_{4}$ (IIb), accompanied by a change in dominant speciation from $\mathbf{1}$ and 2 to $\mathbf{4}$ (or alkene complex 6, Fig. 2). However, even when the ethyl vinyl ether concentration approaches its limiting value $(\approx 10.5 \mathrm{M})$, such saturation may be unachievable. Nonetheless, using more reactive alkenes, other solvents and other temperatures, it may be feasible to deconvolute the interchange (IIa) from the step-wise (IIb) association-dissociation pathways for G-III-Br.

Also of interest is the increased rate of decay of the UV-vis signal from the MLCT band in $\mathbf{1} / \mathbf{2}$, arising by competing oxidation to yield benzaldehyde; a process that appears to be catalysed by trace-metal contaminants. ${ }^{23} \dagger$ Attempts to deliberately induce this effect met with limited success due to unreproducible kinetics and very transient periods of stability of $[\mathbf{1 , 2}]$ prior to decomposition. ${ }^{24}$ Nonetheless, the apparent acceleration of benzylidene metathesis by (catalysed) 


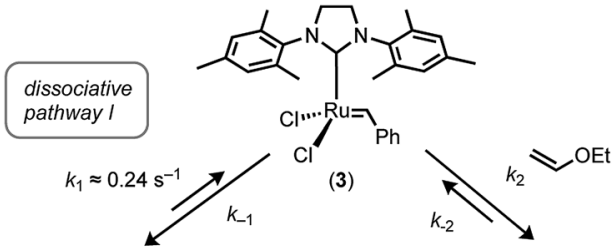<smiles></smiles>

(2)

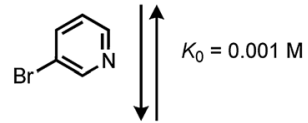

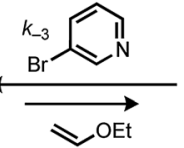

$k_{3} \approx 3 \times 10^{1} \mathrm{M}^{-1} \mathrm{~s}^{-1}$

associative pathway II

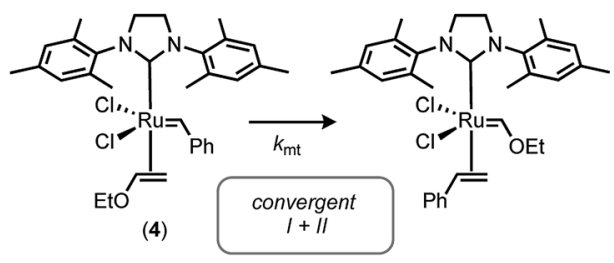

(4)<smiles>Brc1cccnc1</smiles>
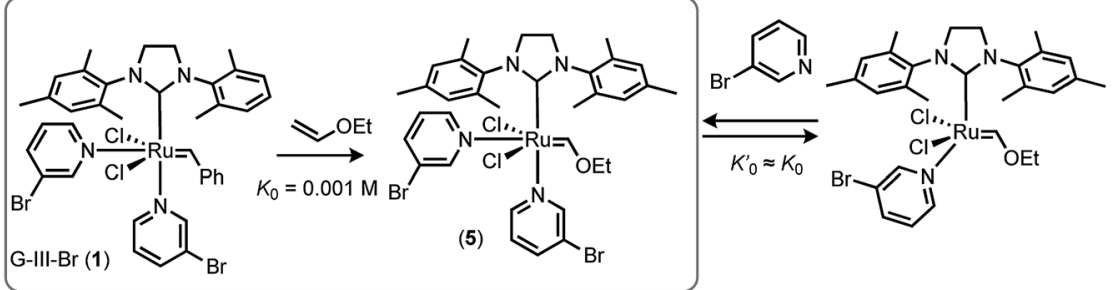

Fig. 10 Summary of overall scheme for reaction of G-III-Br with EVE at $5{ }^{\circ} \mathrm{C}$ in toluene, and minimal kinetic model for satisfactory simulation (see Fig. 8 and 9).

oxidation may account for the previous estimation of the initiation rate ${ }^{19}$ being more than an order of magnitude greatert than that determined herein.

Overall, in the context of optimising the application of the G-III-Br system in catalysis, for a given level of aerobic exposure, conducting reactions at high catalyst and alkene concentrations will favour turnover over oxidative degradation. Moreover, if other alkenes also propagate by the associative pathway, then the selectivity between the competing alkenes may not be a simple linear correlation against their relative concentrations, a factor that may be important in e.g. co-polymerisations.

\section{Experimental}

\section{General}

All reagents were obtained from Sigma Aldrich. Anhydrous organic solvents were obtained from a solvent purification system (MBraun SPS 800) and dispensed

\footnotetext{
\$ The original report (see ESI to ref. 19) indicated that on addition of a solution of G-III-Br in toluene to a solution of EVE in toluene in a cuvette at $5{ }^{\circ} \mathrm{C},\left([\mathrm{Ru}]_{\mathrm{tot}}=0.046 \mathrm{mM}, \mathrm{EVE} 17 \mathrm{mM}\right)$ the UV signal at $354 \mathrm{nM}$ was quenched in less than 0.5 seconds, leading to a dissociative initiation rate $\left(k_{\text {init }}\right)$ of G-III-Br being estimated as $>4 \mathrm{~s}^{-1}$. The rate of reaction of G-III-Br with EVE determined by stopped-flow under the same final concentrations $\left([\mathrm{Ru}]_{\mathrm{tot}}=0.046 \mathrm{mM}\right.$, EVE $\left.17 \mathrm{mM}\right)$ is $0.14 \mathrm{~s}^{-1}$; Fig. 9; see also Table 1, entry 24; $\mathrm{EVE}=20 \mathrm{mM}$.
} 
using gas-tight syringes under a positive pressure of nitrogen. The NMR spectra were recorded at $27^{\circ} \mathrm{C}$ on a Bruker Ascend 400 spectrometer. The ${ }^{1} \mathrm{H}$ and ${ }^{13} \mathrm{C}\left\{{ }^{1} \mathrm{H}\right\}$ spectra were referenced to TMS. The commercial samples of complex 1 (G-III-Br) were found to be of variable quality. For reproducible kinetic data, the complex was prepared from G-II employing the procedure of Grubbs and co-workers. ${ }^{19}$ Thus, G-II (0.5 g, $0.6 \mathrm{mmol})$ and 3-bromopyridine (1.1 mL, $11.0 \mathrm{mmol})$ were added to a $20 \mathrm{~mL}$ vial. The vial was capped and the reaction mixture was left stirring for 5 minutes while the color changed from red to bright green. Pentane $(20 \mathrm{~mL})$ was layered onto the green solution at room temperature to initiate the precipitation of a green solid. The vial was then capped and left at $-20{ }^{\circ} \mathrm{C}$ overnight (freezer). The green precipitate was collected by vacuum-filtration and washed with $4 \times$ $10.0 \mathrm{~mL}$ of room temperature pentane. After drying the precipitate under vacuum, complex 1 was obtained as a green powder $(0.4 \mathrm{~g}, 80 \%$ yield $)$ matching all the reported spectral data. ${ }^{19}$

\section{Kinetic analyses}

The stopped-flow UV-vis experiments were carried out with a Hi-Tech Scientific SFA-20 accessory coupled through a thermostatted umbilical to a Hellma Analytics fused-silica flow cell $\left(5.0 \pm 0.1{ }^{\circ} \mathrm{C}, 80 \mu \mathrm{L}\right.$ volume, with $10 / 2 \mathrm{~mm}$ light paths, and integral mixer), and the outlet connected via the umbilical to a trigger-syringe with microswitch. Within an $\mathrm{N}_{2}$-filled glovebox, stock solutions of G-III-Br (92 mM) and ethyl vinyl ether ( 0.0008 to $1.66 \mathrm{M}$; plus 3-bromopyridine $0-4 \mathrm{mM}$ ) in toluene were prepared in volumetric glassware, then transferred to $10 \mathrm{~mL}$ gas-tight syringes (VWR) coupled to a $1 \mathrm{~mm}$ OD Tefzel tube and sealed with a plugged flangeless PTFE nut. Prior to kinetic experiments the system was washed through with large volumes of toluene, and then a background UV-vis spectrum recorded. The reagents were then loaded into the two independent $2.5 \mathrm{~mL}$ syringe reservoirs via three-way PTFE valves on the SFA-20 connected to the $10 \mathrm{~mL}$ gas tight syringes via flangeless fittings, and using hydraulic overpressure for all liquid transfers, i.e. 'pushing' rather than 'pulling' solutions. The system was then flushed twice with the stock solutions ( $5 \mathrm{~mL}$ of each, in parallel) to ensure complete purging of toluene, before reloading the reservoirs and conducting a series of 10-25 shots, each comprising $0.1 \mathrm{~mL}$ of each stock solution. The shots were initiated by a pneumatic drive at constant 5 bar pressure. The UV spectra were recorded on OceanOptics USB4000 and Flame Spectrometers connected via the cuvette holder to a DH2000-BALUV lamp using solarised resistant grade optical fibres. The data collection was timed by the microswitch on the terminus of the trigger syringe transit, sending a stabilised 5V signal to the spectrometer and PC. 200 UV-vis spectra were recorded over $8-80 \mathrm{~s}$, depending on $k_{\text {obs. }}$, after a dead-time of approximately $8 \mathrm{~ms}$, with a $284-890 \mathrm{~nm}$ spectral window. The UV-vis spectra were analysed with Kinetic Studio software, version 5.02, using singular value decomposition (SVD) and recombination of the first two or three components to filter noise. For G-III, the rates of reaction $\left(k_{\text {obs. }}\right)$ were obtained by non-linear regression of the exponential decay of the signal $\left(A_{t}\right)$ at $354 \mathrm{~nm}$, averaged across a $0.6 \mathrm{~nm}$ spectral range ( 3 data points) from $A_{0}$ to $A_{\infty}$. The data for G-I were obtained analogously, but by non-linear regression of the growth of the signal $\left(A_{t}\right)$ at $484 \mathrm{~nm}$. 


\section{Steady-state rate approximations}

The rate equations for pathway 1, pathway II, and pathways I + II were derived using standard Bodenstein approximations to solve for the mole fraction of premetathesis complex $\mathbf{4}$, as a function of 2 , assuming that the ruthenium species $\mathbf{2}$, accumulating product $\mathbf{5}$ (in mono and bis-pyridyl forms) and bispyridyl complex 1, are dominant over all other complexes. Fitting of eqn (6) (Fig. 11) against the observed data (Table 1) was conducted by non-linear regression of $k_{\text {calc. against }}$ $k_{\text {obs. }}$ using the Excel solver function to minimise the weighted sum square error, SSE $=\Sigma\left\{\left(k_{\text {calc. }}-k_{\text {obs. }}\right)^{2} / k_{\text {obs. }}\right\}$ with $c, d, e, f, g$, and $k_{1}$ as variables. Although the inclusion of $g$ in the fitting gives a marginally better correlation the model is relatively insensitive to $g \leq 5 \times 10^{5}$. A standard quadratic analysis of the mono/ bis-pyridyl speciation from rapid equilibrium $\left(K_{0}\right)$ between $1+5$ and 2 , as dictated by $[\mathrm{Ru}]_{\mathrm{tot}}$ and free $[\mathrm{L}]$, was used to correct the rates for mole fraction $2\left(x_{2}\right.$, see eqn (1, 7 and 8), Fig. 11). The speciation for the mono and bis-pyridyl forms of product 5 was assumed to be similar, i.e. $K_{0}^{\prime} \approx K_{0}$, and thus free $[\mathrm{L}]_{\text {tot }}$ is constant through the reaction.

$$
\begin{aligned}
& k_{\mathrm{obs}} \approx x_{2} k_{\mathrm{mtl}}[4] /[2] ; \text { where } x_{2}=[2] /[\mathrm{Ru}] \\
& {[4] \approx \frac{k_{2}[3][E]+k_{3}[2][E]}{k_{-2}+k_{-3}[L]+k_{\mathrm{mt}}}} \\
& {[3] \approx \frac{k_{1}[2]+k_{-2}[4]}{k_{-1}[L]+k_{2}[E]}} \\
& {[4] \approx \frac{[2]\left(k_{1} k_{2}[E]+k_{-1} k_{3}[L][E]+k_{2} k_{3}[E]^{2}\right)}{k_{-1} k_{-2}[L]+k_{-1} k_{-3}[L]^{2}+k_{2} k_{-3}[L][E]+k_{-1} k_{m t}[L]+k_{2} k_{m t}[E]}} \\
& k_{\mathrm{obs}} \approx x_{2} \frac{1+\frac{k_{-1} k_{3}[\mathrm{~L}]}{k_{1} k_{2}}+\frac{k_{3}[\mathrm{E}]}{k_{1}}}{\frac{[\mathrm{L}]}{k_{1} K_{2} k_{\mathrm{mt}}[\mathrm{E}]}+\frac{k_{-1} k_{-3}[\mathrm{~L}]}{k_{1} k_{2} k_{\mathrm{mt}}[\mathrm{E}]}+\frac{k_{-3}[\mathrm{~L}]}{k_{1} k_{\mathrm{mt}}}+\frac{[\mathrm{L}]}{k_{2} K_{1}[\mathrm{E}]}+\frac{1}{k_{1}}} \\
& k_{\mathrm{obs}} \approx x_{2} \frac{1+c[\mathrm{~L}]+d[\mathrm{E}]}{e[\mathrm{~L}] /[\mathrm{E}]+f[\mathrm{~L}]+g[\mathrm{~L}]^{2}[\mathrm{E}]+1 / k_{1}} \\
& {[L]=[2]+[L]_{\text {exog }}} \\
& {[2]=0.5 \times\left(\sqrt{\left([L]_{\text {exog. }}+K_{0}\right)^{2}-\left(4[R u] K_{0}\right)}-\left([L]_{\text {exog. }}+K_{0}\right)\right)} \\
& x_{2} \approx \frac{1}{1+\left([\mathrm{L}]_{\text {exog. }} \cdot / K_{0}\right)}
\end{aligned}
$$

Fig. 11 Derivation of steady-state approximation for initiation mechanism in which dissociative and interchange pathways (I and Ila) converge at intermediate 4 , in which 4 has a discreet lifetime and can revert to starting materials or proceed to metathesis $\left(k_{\mathrm{mt}}\right)$. An analogous analysis for dissociative and associative pathways (I and $I \mathrm{lb})$ affords essentially the same simplified rate expression as in eqn (6), except that $(1+h)$ replaces $(1)$ in the numerator. 


\section{Conflicts of interest}

There are no conflicts to declare.

\section{Acknowledgements}

The research leading to these results received funding from the European Research Council under the European Union's Seventh Framework Programme (FP7/2007-2013)/ERC Grant Agreement 340163, the University of Edinburgh (V. F.), and the Swiss National Science Foundation (A.G.D.). We thank Edward J. King (TgK Scientific) for extensive technical advice and assistance.

\section{Notes and references}

1 For a recent review on the advances in ruthenium alkylidene catalysed alkene metathesis, see: O. M. Ogba, N. C. Warner, D. J. O'Leary and R. H. Grubbs, Chem. Soc. Rev., 2018, 47, 4510-4544.

2 M. Jeschek, R. Reuter, T. Heinisch, C. Trindler, J. Klehr, S. Panke and T. R. Ward, Nature, 2018, 537, 661-668.

3 For examples of the use of transition metal catalysed alkene metathesis in industrial settings see: D. Stoianova, A. Johns and R. Pederson, Handbook of Metathesis, ed. R. H. Grubbs and A. G. Wenzel, Wiley-VCH Verlag GmbH \& Co. KGaA, 2nd edn, 2015, vol. 1, pp. 699-726, see also ref. 1.

4 For an overview of the mechanistic events in Ru-catalysed metathesis, see: D. J. Nelson, S. Manzini, C. A. Urbina-Blanco and S. P. Nolan, Chem. Commun., 2014, 50, 10355-10375.

5 For an excellent review on the initiation in Ru-catalysed metathesis, see: J. R. Griffiths and S. D. Diver, Handbook of Metathesis, ed. R. H. Grubbs and A. G. Wenzel, Wiley-VCH Verlag GmbH \& Co. KGaA, 2nd edn, 2015, vol. 1, pp. 273-303.

6 For an example of circumventing poor initiation rate in polymerisation, see J. C. Foster, S. Varlas, L. D. Blackman, L. A. Arkinstall and R. K. O’Reilly, Angew. Chem., Int. Ed., 2018, 57, 10672-10676, and references therein.

7 For recent examples of inhibition, see: G. A. Bailey, M. Foscato, C. S. Higman, C. S. Day, V. R. Jensen and D. E. Fogg, J. Am. Chem. Soc., 2018, 140, 6931-6944, and references therein.

8 J. S. Kingsbury, J. P. A. Harrity, P. J. Bonitatebus and A. H. Hoveyda, J. Am. Chem. Soc., 1999, 121, 791-799.

9 For an example of a 'boomerang' system, see: J. M. Bates, J. A. M. Lummiss, G. A. Bailey and D. E. Fogg, ACS Catal., 2014, 4, 2387-2394, and references therein.

10 See e.g. J. A. Love, M. S. Sanford, M. W. Day and R. H. Grubbs, J. Am. Chem. Soc., 2003, 125, 10103-10109.

11 Y. Kim, C.-H. Chen and C. Hilty, Chem. Commun., 2018, 54, 4333-4336.

12 Q. T. Easter and S. A. Blum, Angew. Chem., Int. Ed., 2018, 57, 12027-12032.

13 M. S. Sanford, M. Ulman and R. H. Grubbs, J. Am. Chem. Soc., 2001, 123, 749750.

14 E. L. Dias, S. T. Nguyen and R. H. Grubbs, J. Am. Chem. Soc., 1997, 119, 38873897. 
15 Y. Minenkov, G. Occhipinti and V. R. Jensen, Organometallics, 2013, 32, 20992111.

16 V. Thiel, M. Hendann, K.-J. Wannowius and H. Plenio, J. Am. Chem. Soc., 2012, 134, 1104-1114.

17 I. W. Ashworth, I. H. Hillier, D. J. Nelson, J. M. Percy and M. A. Vincent, Chem. Commun., 2011, 47, 5428-5430.

18 S. B. Garber, J. S. Kingsbury, B. L. Gray and A. H. Hoveyda, J. Am. Chem. Soc., 2000, 122, 8168-8179.

19 J. A. Love, J. P. Morgan, T. M. Trnka and R. H. Grubbs, Angew. Chem., Int. Ed., 2002, 41, 4035-4037.

20 B. Trzaskowski and K. Grela, Organometallics, 2013, 32, 3625-3630.

21 D. J. Walsh, S. H. Lau, M. G. Hyatt and D. Guironnet, J. Am. Chem. Soc., 2017, 139, 13644-13647.

22 A. B. Chang, T.-P. Lin, N. B. Thompson, S.-X. Luo, A. L. Liberman-Martin, H.-Y. Chen, B. Lee and R. H. Grubbs, J. Am. Chem. Soc., 2017, 139, 1768317693.

23 I. Thomé, A. Nijs and C. Bolm, Chem. Soc. Rev., 2012, 41, 979-987.

24 S. Guidone, O. Songis, F. Nahra and C. S. J. Cazin, ACS Catal., 2015, 5, 26972701. 